\title{
Tumor gene therapy by MVA-mediated expression of T-cell-stimulating antibodies
}

\author{
Stephane Paul, ${ }^{1}$ Etienne Regulier, ${ }^{2}$ Ronald Rooke, ${ }^{1}$ Fabienne Stoeckel, ${ }^{1}$ Michel Geist, ${ }^{2}$ \\ Horst Homann, ${ }^{2}$ Jean-Marc Balloul, ${ }^{2}$ Dominique Villeval, ${ }^{2}$ Yves Poitevin, ${ }^{2}$ \\ Marie-Paule Kieny, ${ }^{3}$ and R Bruce Acres ${ }^{1}$
}

${ }^{1}$ Clinical and Experimental Immunology Laboratory; ${ }^{2}$ Molecular Immunology Laboratory, Transgene, Strasbourg 67082, France; and ${ }^{3}$ INSERM U74, Strasbourg 67000, France.

Immune responses to tumor-associated antigens are often dampened by a tumor-induced state of immune anergy. Previous work has attempted to overcome tumor - induced T - cell anergy by the direct injection of vectors carrying the genes encoding one of a variety of cytokines. We hypothesised that the polyclonal stimulation of T cells, preferably through the TCR complex, would result in a cascade of cytokines associated with T-cell activation and would be best able to overcome T-cell anergy. Here we use the highly attenuated MVA poxvirus to express on tumor cells, in vitro and in vivo, either of three membrane-bound monoclonal antibodies specific for murine TCR complex. Using this system, we have expressed antibodies specific for the CD3 $\varepsilon$ chain (KT3), TCR $\alpha / \beta$ complex (H57 597 ), and $V \beta 7$ chain (TR310). Tumor cells bristling with these antibodies are capable of inducing murine T-cell proliferation and cytokine production. When injected into growing tumors (P815, RenCa, and B16F10), these constructs induce the activation of immune effector cells and result in the rejection of the tumor. Histological and FACS analysis of tumor-infiltrating leukocytes reveal that the injection of recombinant virus-expressing antibodies specific for the TCR complex attracts and activates $\left(\mathrm{CD} 25^{+}, \mathrm{CD} 9^{+}\right)$ CD4 and CD8 lymphocytes. This approach represents a novel strategy to overcome T-cell anergy in tumors and allow the stimulation of tumor-specific T cells.

Cancer Gene Therapy (2002) 9, 470-477 DOI: 10.1038/sj/cgt/7700461

Keywords: recombinant antibodies; T cell; MVA; cancer immunotherapy; anti-CD3

$\mathrm{C}$ urrent efforts to develop immunotherapy of cancer focus on the induction of tumor-specific anamnestic immune response. Several strategies have been developed to recruit specific effector $\mathrm{T}$ cells towards tumors and to stimulate them locally. Bispecific antibodies with specificities for the CD3 molecule on T cells and tumor cell surface antigen can target effector $\mathrm{T}$ cells towards tumor cells. ${ }^{1}$ Limitations with this approach are that the identity of the tumor antigen is required and that intravenous administration of bispecific antibodies can induce systemic cytokine release and toxicity. ${ }^{2}$ The use of cytokines exhibiting pleiotropic activities has also been investigated in order to circumvent the immune anergy associated with many cancers and to promote a tumor-specific T-cell-mediated immune response. ${ }^{3}$ Because systemic delivery of high doses of cytokines is toxic, in situ expression of cytokine genes in solid tumors is considered preferable. ${ }^{4}$ However, therapeutic effects resulting from the overexpression of a single cytokine are often limited and depend upon natural killer or other nonantigen-specific cellular effectors. ${ }^{4}$

One interesting avenue is to use the cytokine cascade associated with strong antigenic stimulation of T cells. This can be achieved with monoclonal antibodies (mAbs)

Address correspondence and reprint requests to: Stephane Paul, Clinical and Experimental Immunology Laboratory, Transgene, Strasbourg 67082, France.E-mail: paul@transgene.fr recognizing certain $\mathrm{T}$-cell surface molecules (i.e., TCR, CD3, CD2) that mimic antigenic stimulation of T cells. ${ }^{5}$ The relevance of this approach was demonstrated when tumor-bearing mice rejected their tumors following in situ injection of an anti-CD3 antibody. ${ }^{6}$ Also, tumor cells have been engineered to express anti-CD3 antibodies by in vitro transduction. ${ }^{7,8}$ Murine tumor cells engineered to express anti-CD3 antibodies prior to implantation induce a T-cell-mediated control of tumor growth. ${ }^{7}$

\section{Materials and methods \\ Antibodies and cytokines}

FITC-labeled or unconjugated mouse anti-rat IgG, rat antihamster IgG, and anti-rat kappa light chain were purchased from Jackson ImmunoResearch Laboratories (Pennsylvania, PA) and used for FACS or Western blot detection of TCRspecific mAbs. FITC or phycoerythrin-labeled rat antimouse CD4, CD8, CD3, CD69, MAC1, CD11c, and $\mathrm{Ia}^{\mathrm{b}}$ or unconjugated rat anti-mouse CD4 and CD8 were used as defined by the manufacturer (Pharmingen, San Diego, CA). Unconjugated rabbit anti-human CD3 (which crossreacts with mouse CD3 ) or rabbit anti-rat IgG and peroxidaselabeled goat antirabbit were used at concentrations suggested by Dako (Trappes, France).

Recombinant human IL-2 was purchased from R\&D Systems (Abingdon, UK). Concanavalin A (ConA) was 
used at $1 \mu \mathrm{g} / \mathrm{mL}$ and purchased from Sigma-Aldrich (Saint Quentin Fellavier, France).

Tumor cells. P815 murine mastocytoma (DBA/2; $\mathrm{FcR}^{+}$, $\left.\mathrm{H}_{2} \mathrm{D}^{\mathrm{d}}, \mathrm{MHCI}^{+}, \mathrm{ICAM}^{+}, \mathrm{CD} 48^{+}\right)$and B16F10 (C57B1/6; $\mathrm{H}_{2} \mathrm{D}^{\mathrm{b}}, \mathrm{MHCI}^{-}, \mathrm{MHCII}^{-}, \mathrm{ICAM}^{-}, \mathrm{CD}^{-} 8^{-}$) murine melanoma cancer cell lines were obtained from the American Type Culture Collection (ATCC; Manassas, VA). RenCa murine renal carcinoma (BALB-C; H2D ${ }^{\mathrm{d}}, \mathrm{MHCI}^{+}$, $\mathrm{MHCII}^{+}, \mathrm{Fas}^{+}$) cell line was the generous gift of Dr. G Hillman (University of Chicago). BHK-21 cells were also provided by ATCC (Rockville, MD). All cell lines were tested negative for mycoplasma using Hoechst dye, cell culture, and polymerase chain reaction.

Hybridoma. TR310 (rat anti-mouse V $\beta 7$; IgG2b) and H57597 hybridomas (hamster anti-mouse $\operatorname{TCR} \alpha \beta$; IgG) cell lines were obtained from the ATCC. KT3 hybridoma (rat anti-mouse CD3 $\varepsilon$; IgG2a) was generously provided by Dr. D Mathis (Harvard Medical School, Boston, MA).

Recombinant viruses. Recombinant viruses were constructed as reported elsewhere. ${ }^{9}$ Briefly, the early-late vaccinia virus promoters $\mathrm{pH} 5 \mathrm{R}^{10}$ and $\mathrm{p} 7.5^{11}$ were isolated from vaccinia virus WR DNA by polymerase chain reaction. Heavy - and light-chain cDNA of the antibodies were synthesized from total RNA extracted from KT3, H57-597, and TR310 hybridomas using the Universal Riboclone cDNA Synthesis System (Promega, Madison, WI) and cloned into a pBlue- script vector for sequencing. The heavy chain of each mAb was cloned in phase upstream of the sequence coding for the transmembrane and intracytoplasmic domains of the rabies glycoprotein (Fig 1). ${ }^{12,13}$ The cDNA for the native light and modified heavy chains were introduced into MVA transfer plasmid vector downstream of $7.5 \mathrm{kDa}$ or ph5r vaccinia promoters, in reverse orientations similar to that described previously. ${ }^{9}$

Generation of MVATG14205 (TR310), MVATG14237 (H57), and MVATG14240 (KT3) was performed by homologous recombination in primary chicken embryo fibroblasts using pTG14205, pTG14237, and pTG14240 MVA transfer plasmid (Fig 1). ${ }^{9}$ Purification of viral stocks generated viral suspensions devoid of recombinant mAbs (data not shown).

\section{Detection of TCR-specific mAbs expression}

Antibody expression was analysed by Western blot according to the ECL ${ }^{\text {IIII }}$ Western blotting protocol provided by Amersham Life Sciences (Amersham, UK). Primary chicken embryo fibroblasts were infected at a multiplicity of infection (MOI) of 1 . Twenty - four hours after infection, the cells were washed once with PBS and disrupted in sample buffer (Novex) by sonication. Homogenates were heated for 3 minutes at $95^{\circ} \mathrm{C}$ prior to fractionation on a $13 \%$ SDS polyacrylamide gel. Fractioned proteins were transferred from the gel to a PVDF membrane. The TCR-specific

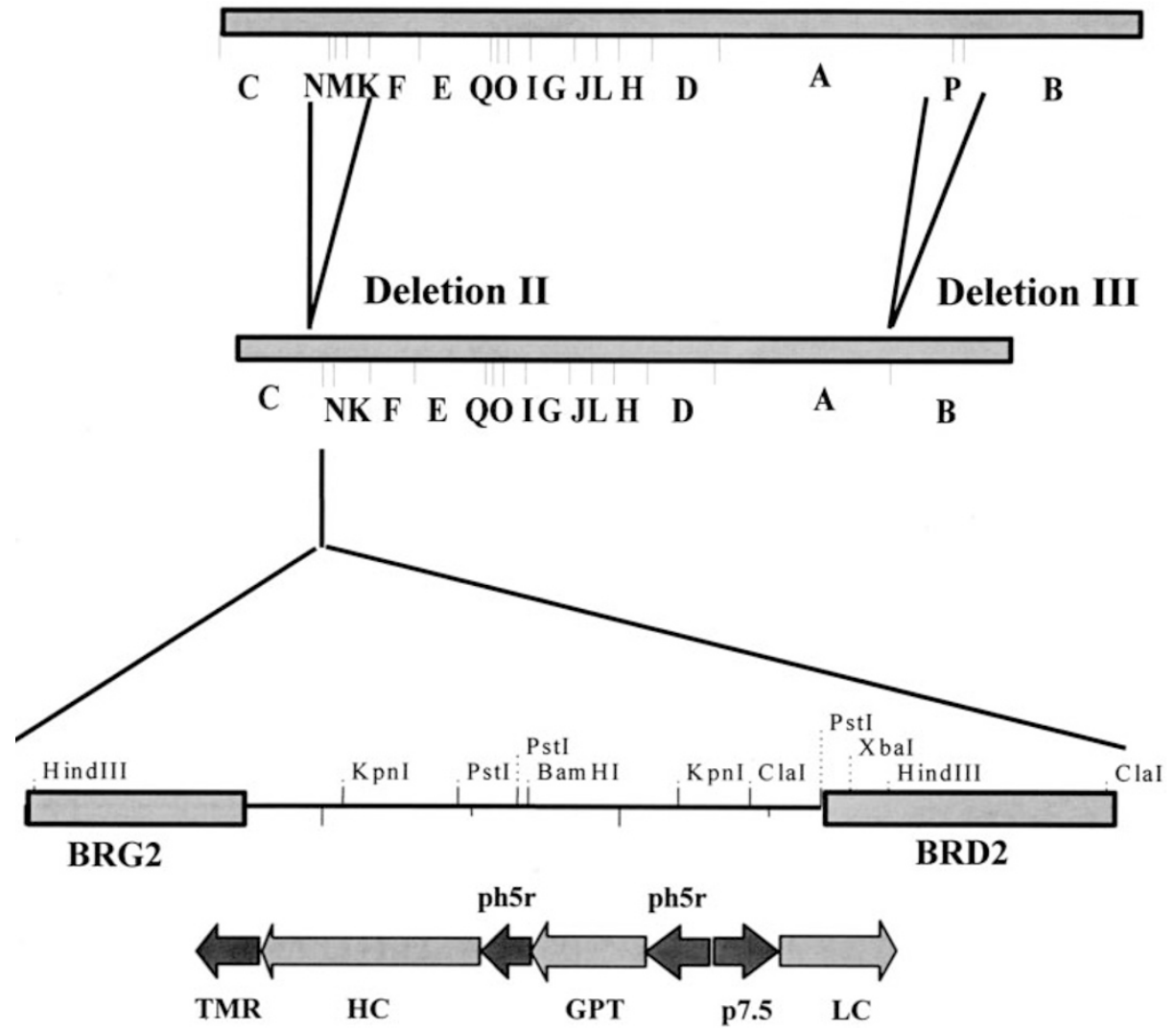

CVA wild-type $208 \mathrm{~kb}$

MVAN33 177 kb

Figure 1 Schematic representation of recombinant MVA. CVA: parental Copenhagen virus A; BRG 2 and BRD2: flanking sequences surrounding deletion II of MVA; alphabetic characters correspond to HindlII fragments of MVA genome; ph5r: early-late vaccinia virus promoter; p7.5: earlylate vaccinia virus promoter; GPT: Escherichia coli xanthine-guanine phosphoribosyltransferase selection gene; TMR: sequence coding for the transmembrane and intracytoplasmic domains of rabies glycoprotein; HC: heavy-chain cDNA; LC: light-chain cDNA. 
mAbs were probed using biotinylated anti-IgG and revealed with horseradish peroxidase-labeled streptavidin (10 $\mu \mathrm{g} / \mathrm{mL})$.

TCR-specific mAbs expression in primary chicken embryo fibroblasts was also analysed by FACS using FITC-labeled goat anti-rat or mouse anti-hamster IgG.

MVA infection of cells for flow cytometry analysis. BHK-21 cells were infected overnight at an MOI of 0.1 . The following day, cells were washed and assessed for expression of membrane bound antibody as described elsewhere. ${ }^{9}$

$T$-cell proliferation assay. Mouse spleen cell proliferation was assessed by the uptake of $\left[{ }^{3} \mathrm{H}\right]$ thymidine. Target tumor cells (RenCa or B16F10) were infected at an MOI of 0.5 for 12 hours then treated with mitomycin C ( $50 \mu \mathrm{g} / \mathrm{mL}$; Sigma ) for 1 hour. After washing, infected tumor cells and splenocytes were co-cultured at the ratios indicated. As positive control, spleen cells $\left(5 \times 10^{4}\right.$ cells/well $)$ were stimulated in complete medium with either ConA (10 $\mu \mathrm{g} /$ $\mathrm{mL}$ ) or $100 \mathrm{ng} / \mathrm{mL}$ recombinant murine IL-2 (R\&D Systems). After 96 hours, the cells were pulsed with $1 \mu \mathrm{Ci}$ / well $\left[{ }^{3} \mathrm{H}\right]$ thymidine. Incorporation of $\left[{ }^{3} \mathrm{H}\right]$ thymidine into the DNA of proliferating T cells was measured by harvesting cellular DNA onto glass filter paper (PHD Harvester; Cambridge Technology, Cambridge, UK) after 4 hours and by counting the radioactivity in a liquid scintillation counter (Beckman Coulter, Roissy, France). All measurements were in triplicate.

Measurement of supernatant cytokine content. P815 cells were infected at an MOI of 0.5 for 12 hours then treated with mitomycin C ( $50 \mu \mathrm{g} / \mathrm{mL}$; Sigma) for 1 hour. After washing, infected tumor cells and splenocytes were co-cultured at a 1:10 ratio. As positive control, spleen cells $\left(5 \times 10^{4}\right.$ cells/ well) were stimulated in complete medium with ConA (10 $\mu \mathrm{g} / \mathrm{mL})$. As negative control, spleen cells were co-cultured for 24 hours with P815 cells infected with MVAN33 or preincubated with an irrelevant isotype-matched antibody ( $50 \mu \mathrm{g} / \mathrm{mL})$. Culture supernatants were then tested for IL-4 or IFN- $\gamma$ content using specific immunoassay (Quantikine; R\&D Systems ) according to the manufacture's instructions. All measurements were in triplicate.

In vivo experiments. The mouse P815, B16F10, and RenCa tumors were established by injecting $100 \mu \mathrm{L}$ of PBS containing $3 \times 10^{5}$ cells subcutaneously into the flanks of 6-week-old female immunocompetent B6D2 F1 mice (IFFA CREDO, Lyon, France). Eight days later, when tumors were palpable, the mice were randomized (10 mice per group) and their tumors were injected with the indicated MVA vectors as follows. Viruses were resuspended in $10 \mathrm{mM}$

A
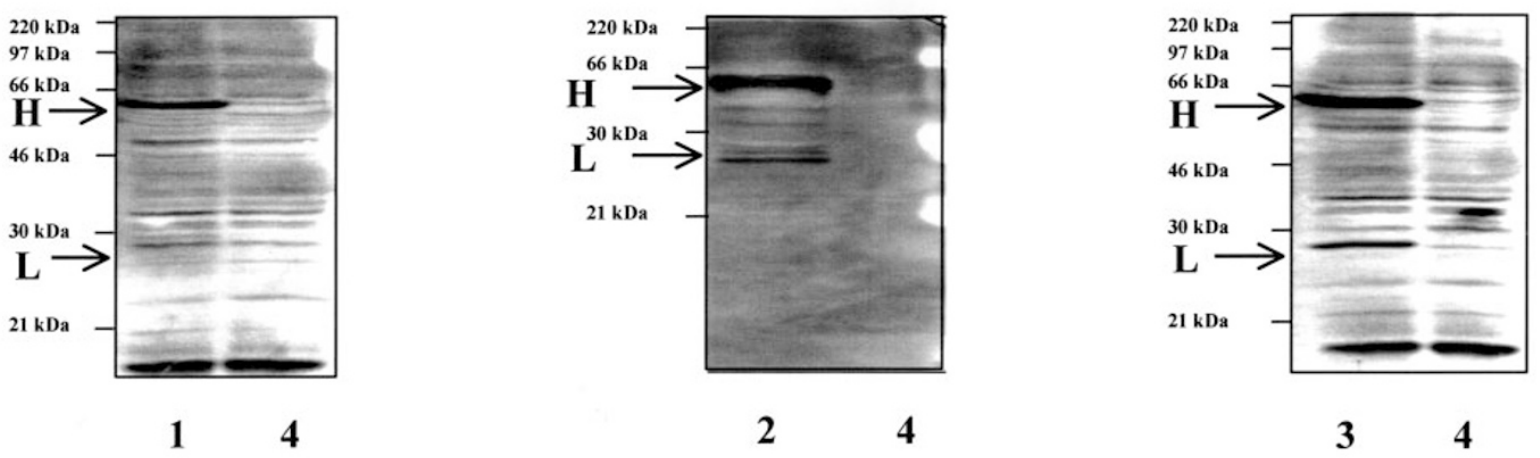

B
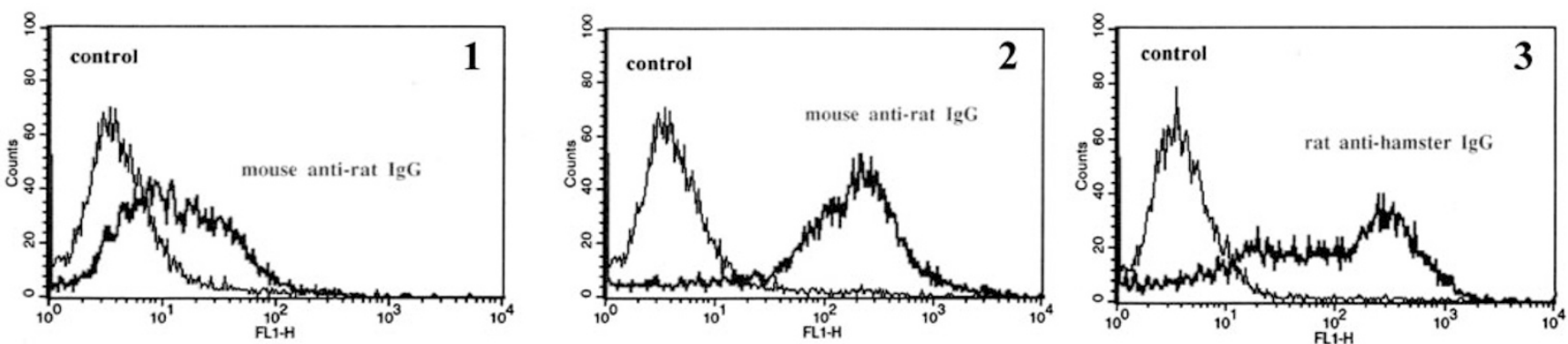

Figure 2 In vitro functionality of MVA recombinants expressing TCR/CD3-specific antibodies. A: Western blot analysis of extracts of BHK-21 cells infected with MVA-TR310 (1), MVA-H57 (2), MVA-KT3 (3), or with control virus (MVAN33, 4). Molecular weight markers are indicated on the left. Heavy $(\mathrm{H})$ and light $(\mathrm{L})$ chains are indicated. B: Flow cytometry analysis of TCR/CD3-specific antibodies expression on BHK-21 infected by MVA-TR310 (1), MVA-KT3 (2), or MVA-H57 (3). 
Tris $-\mathrm{HCl}, \mathrm{pH} 7.5,10 \mathrm{mM} \mathrm{MgCl}_{2}$ at a concentration of $2 \times 10^{7} \mathrm{pfu} / 100 \mu \mathrm{L}$, and injected into the tumors on days 8 , 10 , and 12. Tumor sizes were measured using calipers. Tumor volumes were calculated in cubic millimeter using the formula: $(\pi / 6)($ length $\times$ width $\times$ height $)$.

Statistical analyses. Statistical analyses were performed using the log rank application for Kaplan-Meir survival curves and the $U$ test of Mann-Whitney for tumor volume curves (Statistica 5.1 software; StatSoft). A $P<.05$ was considered statistically significant.

Immunohistochemistry or flow cytometry analysis of in vivo response. Tumors were established and injected with the various viruses as described above for in vivo experiments. On day 13, tumors were measured and excised. For flow cytometry analysis, tumors were disrupted by collagenase (Sigma) digestion, cells were stained with the indicated antibodies, and population analysed by cytofluorimetry. ${ }^{14}$ For immunohistochemistry, tumors were fixed in $10 \%$ neutral formalin. The samples were embedded and sectioned to $5 \mu \mathrm{m}$. After rinsing and fixing with acetone, slides were incubated with primary antibodies. After washing, slides were incubated with the secondary rabbit anti-rat immunoglobulin. Slides were then rinsed and incubated with a peroxidase-conjugated goat anti-rabbit $\mathrm{Ab}$ for amplification of the response, followed by DAB (diaminobenzidine) color development. Slides were observed at $\times 20$ magnification.

\section{Results}

\section{Construction of MVA expressing T-cell-stimulating antibodies}

We have used the highly attenuated Modified Ankara Virus (MVA) to deliver and express in tumor cells membranebound mAbs specific for murine $\mathrm{CD} 3 \varepsilon$ chain (KT3), ${ }^{15}$ TCR $\alpha / \beta$ complex (H57-597), ${ }^{16}$ or V $\beta 7$ chain (TR310). ${ }^{17}$ In each case, the heavy chain of the antibody is fused, as shown in Figure 1, to the membrane anchoring region of the rabies virus glycoprotein, which has been shown to remain attached to the surface of infected cells. ${ }^{18}$
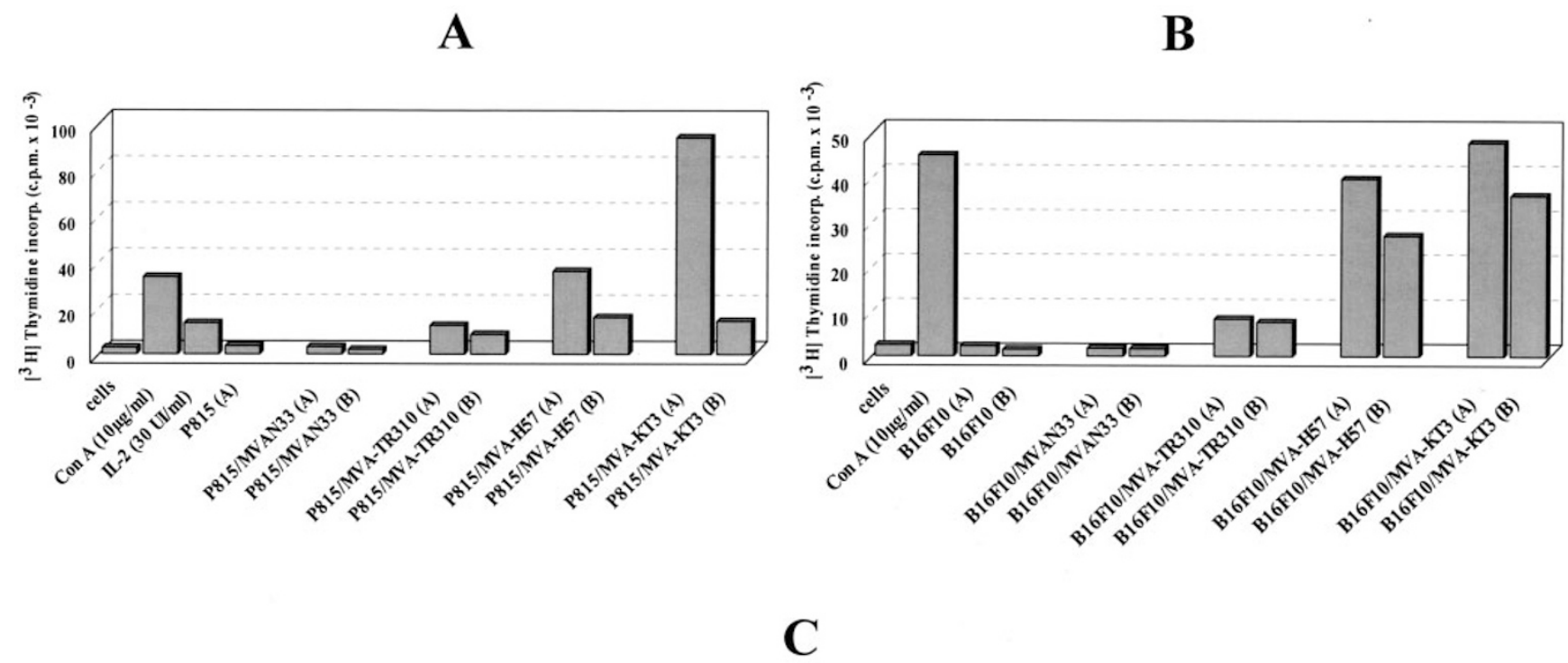

\begin{tabular}{|c|c|c|}
\hline & IL-4 $(\mathrm{pg} / \mathrm{ml})$ & IFN- $\gamma(\mathrm{pg} / \mathrm{ml})$ \\
\hline $\mathrm{conA}$ & 250 & 500 \\
\hline $\mathrm{Ab}$ & $<50$ & 40 \\
\hline MVAN33 & $<50$ & 70 \\
\hline MVA-TR310 & $<50$ & 75 \\
\hline MVA-H57 & 100 & 550 \\
\hline MVA-KT3 & 250 & $>600$ \\
\hline
\end{tabular}

Figure 3 A,B: In vitro T-cell proliferation induced by tumor cells [P815(C); B16F10(D)] infected with the recombinant MVA. As positive control, splenocytes were stimulated with $10 \mu \mathrm{g} / \mathrm{mL}$ ConA or $100 \mathrm{ng} / \mathrm{mL} \mathrm{rmL-2}$. As negative control, tumor cells or control virus-infected tumor cells were used. For each construct, two concentrations of infected tumor cells were evaluated $(A=10,000$ cells $/$ well or $B=1000$ cells $/$ well $)$ with a constant number $\left(1 \times 10^{5}\right)$ of splenocytes per well. Similar results were found in repeat experiments. Standard deviation never exceeded $5 \%$ of reported values (not shown). C: Measurement of mouse IL-4 or IFN- $\gamma$ in the supernatants from co-cultures of splenocytes and infected P815 cells (MVA-TR310, MVA-H57, and MVA-KT3). As positive control, spleen cells were stimulated in complete medium with ConA. As negative controls, spleen cells were co-cultured with P815 cells infected with MVAN33 or preincubated with an irrelevant isotype-matched antibody (Ab). Results are expressed as picograms per milliliter per million effector cells per 24 hours. 
All MVA recombinants were shown to infect tumor cells in vitro, and to promote the expression of mAbs of the correct specificity on the tumor cells (Fig 2, A and B). For MVA-TR310, the band corresponding to the kappa light chain is faint but the expression is also detectable by flow cytometry analysis (data not shown). Cells infected with these constructs, but not with the negative control construct (MVAN33), expressed antibody heavy and light chains that remained membrane-anchored (data not shown). Previous reports have shown that mAbs specific for the T-cell receptor complex were able to stimulate $\mathrm{T}$ cells when bound to a matrix and able to crosslink the TCR complex. This includes binding to beads, to plastic tissue culture vessels, or on antigen-presenting cells through the Fc receptor. ${ }^{19,20}$ In contrast, high doses of soluble anti-CD3 (OKT3) are immunosuppressive. $^{21,22}$

In vitro immune stimulation

We first showed that $\mathrm{Fc}$ receptor-positive $\left(\mathrm{FcR}^{+}\right) \mathrm{P} 815$ murine tumor cells coated with exogenously added purified mAbs (KT3, H57, and TR310) can be used as a matrix to stimulate in vitro $\mathrm{T}$-cell proliferation (data not shown). P815 and B16F10 tumor models were then chosen because of their distinct immunogenicity and genetic background, and were infected with the three MVA recombinants or control MVA. The ability of antibody-expressing cells to induce proliferation of autologous murine spleen cells is clearly shown in Figure 3, A and B). The construct carrying the heavy and light chains for the TR310 mAb (MVA-TR310) was the least effective (Fig 3, A and D). This can be explained by the fact that only $3 \%$ of the entire T-cell repertoire in DBA or $\mathrm{C} 57 \mathrm{Bl} / 6$ expresses $\mathrm{T}$-cell receptors made with the $\mathrm{V} \beta 7$ variable region. ${ }^{17}$ The following experiments were restricted to the anti-TCR $\alpha / \beta$ mAb (MVA-H57) or the CD3 $\varepsilon$ specific mAb (MVA-KT3) constructs. Measurement of IL4 and IFN- $\gamma$ content of supernatants obtained after in vitro immune stimulation indicates evidence of the induction of both Th1 and Th2 T-cell stimulation with both MVA-H57 and MVA-KT3 (Fig 3C).

\section{Effects on in vivo tumor growth}

The consequences of intratumoral expression of either H57 or KT3 on tumor growth and mouse survival in three murine

\section{A (P815)}

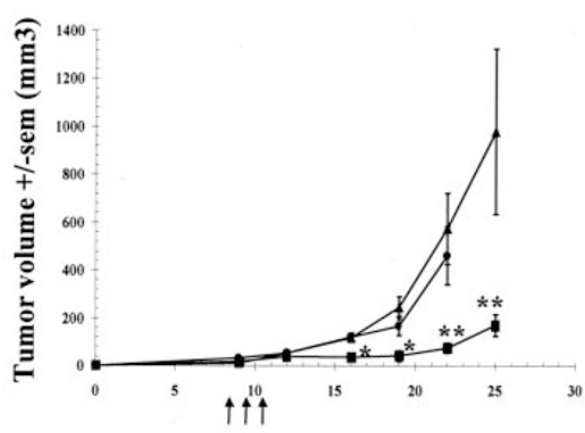

A MVAN33
B (RenCa)

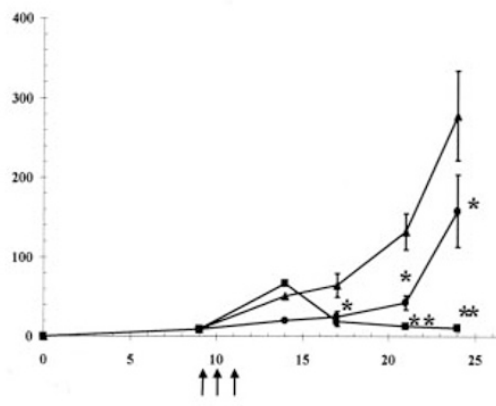

MVA-H57

\section{C (B16F10)}

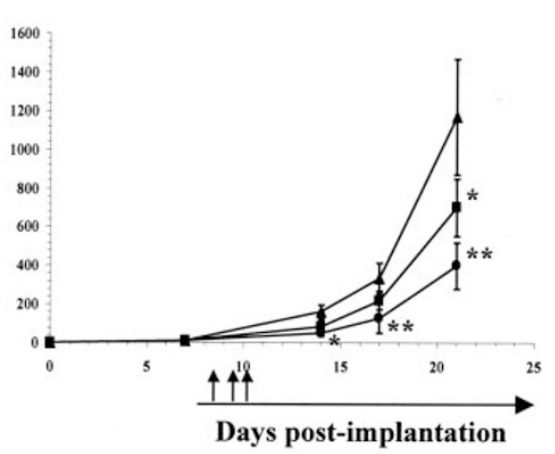

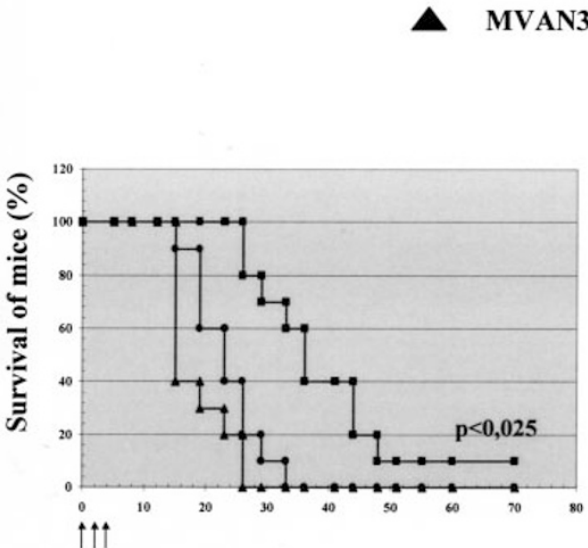
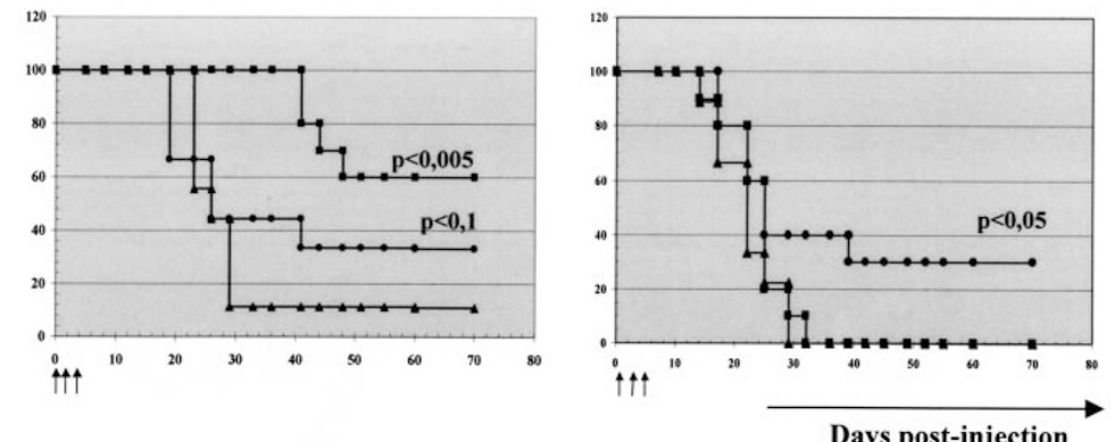

Days post-injection

Figure 4 In vivo anti-tumor activity of MVA expressing TCR-specific antibodies using P815 (A), RenCa (B), and B16F10 (C). Tumors growing in B6D2 mice ( groups of 10) were injected three times with $2 \times 10^{7}$ pfu of control MVAN33 ( $\boldsymbol{\Delta}$ ), MVA-H57 ( $)$ ), or MVA-KT3 ( $\boldsymbol{\square}$ ). Tumor volumes $\left(\mathrm{mm}^{3}\right)$ and survival of mice (\%) were monitored. Significant differences between the mean survival of animals treated with control virus or recombinant MVA are indicated. Statistical values were calculated as described in Materials and Methods and are indicated in the figure: ${ }^{*} P<.05$; ${ }^{* *} P<.005$. Vertical arrows indicate days of virus injection. 
Table 1 Tumor-specific immunity was evaluated in surviving mice after MVA-H57 or MVA-KT3 treatment, by reinjecting the same tumor cells (B16F10 or RenCa) contralaterally 70 days postinitial implantation

\begin{tabular}{lll}
\hline & \multicolumn{1}{c}{ B16F10 } & \multicolumn{1}{c}{ RenCa } \\
\hline MVA-H57 & $3 / 3(100 \%)$ & $1 / 3(33 \%)$ \\
MVA-KT3 & $4 / 4(100 \%)$ & $0 / 3(0 \%)$ \\
& $0 / 0$ & $6 / 6(100 \%)$ \\
& $0 / 0$ & $7 / 7(100 \%)$
\end{tabular}

Survival of mice (\%) was monitored.

tumor models (P815, RenCa, and B16F10) in B6D2 mice were investigated. In vitro experiments have shown that the infection efficiency with recombinant MVA is not the same with these three tumor models. RenCa and B16F10 infect at the same rate $(50 \%$ at an MOI of 0.1$)$, but P815 infects at a rate which is 10 times less (data not shown). Infection rates in vivo are all approximately the same (about $1 \%$ of tumor cells with an injection of $\left.2 \times 10^{7} \mathrm{pfu}\right)$.
Both recombinant vectors clearly influenced tumor growth in each model examined with the exception of MVA-H57 in the P815 model (Fig 4, A-C). This correlates with an increase in the survival time of treated mice. In the RenCa model, $60 \%$ and $33 \%$ of mice injected with MVAKT3 and MVA-H57 completely rejected their tumors, respectively. In the $\mathrm{B} 16 \mathrm{~F} 10$ model, $30 \%$ of tumors was rejected when injected with MVA-H57, whereas MVAKT3 was ineffective. One explanation for this difference could be that antibodies which crosslink the T-cell receptor ( such as H57) induce a different signal transduction pathway in $\mathrm{T}$ cells than antibodies which crosslink the CD3 complex (as does KT3). ${ }^{23}$ Nevertheless, it is unclear at present whether the observed difference in the efficacy of MVA-KT3 and MVA-H57 can be attributed to the nature of the signalling pathway, to inherent immunogenicity of the antibodies (rat versus hamster antibody), to differences in cell surface antigen expression between B16F10 and RenCa cells, or to infection and/or expression capacity of the tumor cells. The construct MVA-TR310 was also tested for tumor immunotherapy in both the P815 and RenCa

A

CD3
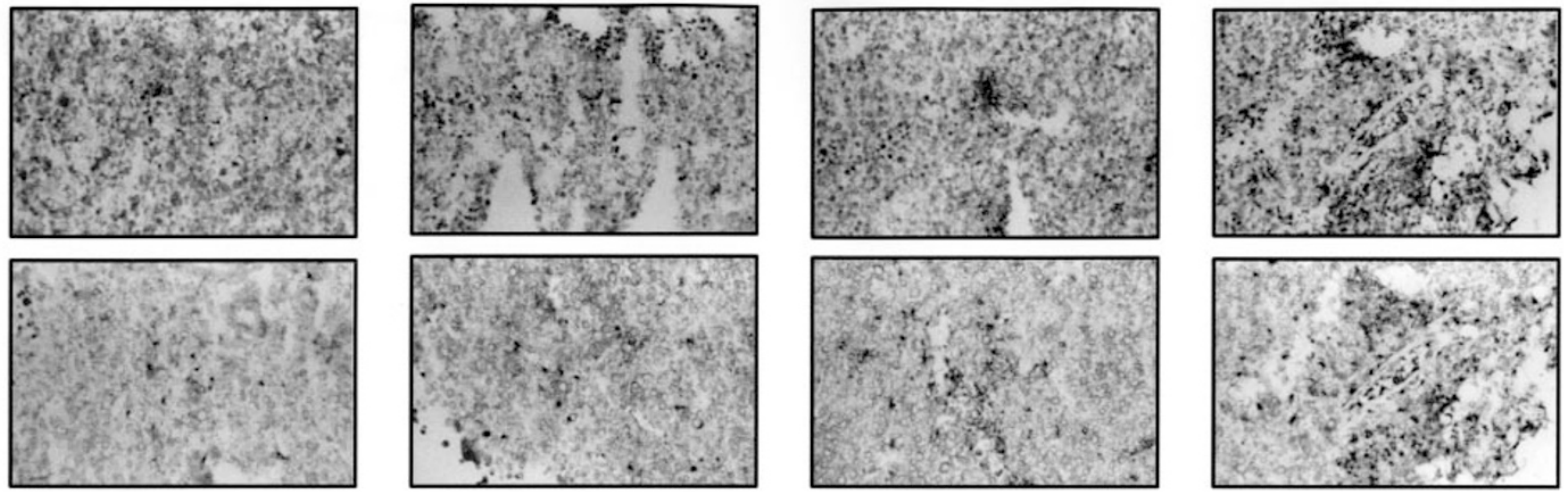

CD4

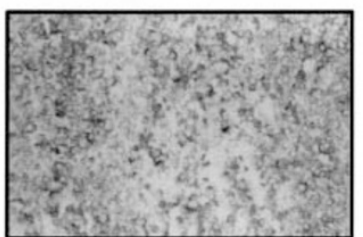

RenCa

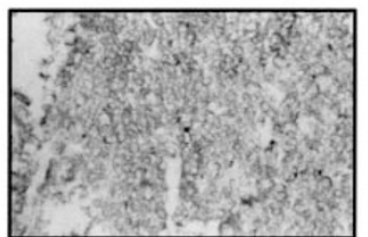

RenCa/MVAN33

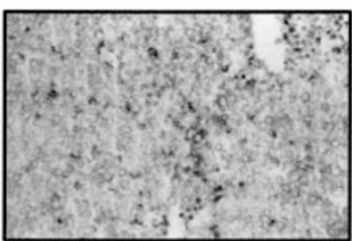

RenCa/MVA-H57

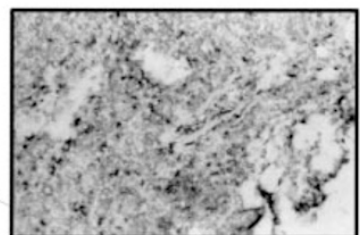

RenCa/MVA-KT3

B

\begin{tabular}{|l|c|c|c|c|}
\hline Infiltrating Cells (\%) & RenCa & MVAN33 & MVA-H57 & MVA-KT3 \\
\hline CD4+ & $0 \pm 0$ & $4,2 \pm 2$ & $9 \pm 3$ & $12 \pm 2$ \\
\hline CD4+/CD25+ & $0 \pm 0$ & $0,7 \pm 0,3$ & $3,8 \pm 1$ & $6,2 \pm 0,4$ \\
\hline CD8+ & $4 \pm 1$ & $11_{ \pm} 1$ & $17 \pm 3$ & $33 \pm 5$ \\
\hline CD8+/CD25+ & $0,2 \pm 0,01$ & $0,9 \pm 0,1$ & $4,5 \pm 1,5$ & $14,5 \pm 3$ \\
\hline CD3+ & $4 \pm 0,2$ & $12,8_{ \pm} 0,2$ & $28,6 \pm 2,5$ & $40 \pm 5$ \\
\hline CD3+/CD25+ & $0,04 \pm 0,01$ & $2,4 \pm 0,3$ & $12 \pm 0,5$ & $17,5 \pm 3$ \\
\hline CD3+/CD69+ & $0,1 \pm 0,01$ & $2,5_{ \pm} 0,1$ & $13,2 \pm 3$ & $22,9 \pm 0,8$ \\
\hline MAC1+ & $11_{ \pm} 2$ & $30_{ \pm} 5$ & $35_{ \pm} 4$ & $37,6 \pm 2$ \\
\hline CD11c+ & $1 \pm 0,1$ & $6 \pm 2$ & $6,7 \pm 0,3$ & $19 \pm 2$ \\
\hline
\end{tabular}

Figure 5 Tumor immune cells infiltration induced by recombinant MVA expressing TCR complex-specific antibodies injection in RenCa tumors. A: Tissue sections were prepared and stained as described (Materials and methods). B: FACScan analysis of single cells suspension prepared from the tumors and stained as described (Materials and methods). Percentages represent the number of positive cells in $1 \times 10^{5}$ ungated total events. Each result is representative of three animals, each with similar results. Standard deviations are indicated. 
models. In both of these model systems, this construct was ineffective, indicating that the inherent immunogenicity of a membrane-bound rat antibody is not sufficient to induce rejection of the tumor (data not shown). All experiments included a group whose tumors were injected with buffer alone. The survival curves of these animals did not differ significantly from that of the groups injected with the negative control virus MVAN33 (data not shown).

Tumor-specific immunity was evaluated in surviving mice by reinjecting the same tumor cells contralaterally 70 days postinitial implantation. Mice treated with MVA-KT3 and having rejected RenCa tumors were all resistant to the second challenge (Table 1). In contrast, only one of three surviving mice treated with MVA-H57 could reject the second RenCa tumor challenge (Table 1). In the B16F10 model, all mice surviving following the MVA-H57 treatment were protected against rechallenge (Table 1). This is in contrast with previously reported experiments in which in vitro transfected tumor cells expressing membrane bound anti-CD3 antibodies prior to implantation resulted in control of tumor growth and did not allow the establishment of a tumor-specific immune memory. ${ }^{7}$

\section{Phenotype analysis of intratumor leukocytes}

In vivo growing RenCa tumors injected with the MVA recombinants were examined for immune cell infiltration by immunohistochemistry at day 5 postviruses injection. Immunohistochemistry analysis (Fig 5A) demonstrated that injection of control MVAN33 attracted some T cells to the tumor, but these were restricted to areas around tumor vascularisation (data not shown). In contrast, tumors injected with MVA-H57 and MVA-KT3 were shown to be infiltrated with both CD4- and CD8-expressing T cells. Cytofluorimetry analysis of extracted tumors showed that these T cells expressed CD25 and CD69, both of which are markers of T-cell activation (Fig 5B). Treatment with either recombinant virus also attracted a greater number of the CD11c ${ }^{+}$dendritic cells found to be mature $\left(\mathrm{CD} 11 \mathrm{c}^{+} / \mathrm{Ia}^{\mathrm{b}+}\right)$ in MVA-KT3-treated tumors. It is noteworthy that MVAKT3 injected into RenCa tumors attracted more infiltrating cells than MVA-H57, which correlates well with their respective efficacy on tumor growth and animal survival, as shown in Figure 4B.

\section{Discussion}

In this report, we have described a novel method for stimulating $\mathrm{T}$ cells in situ by using recombinant MVA expressing membrane-bound T-cell-stimulating mAbs. By applying this approach to models of immunotherapy of solid tumors, we have demonstrated that it results in the attraction of $\mathrm{T}$ cells to the tumor site, some of which have an activated phenotype. This is in contrast to observations using adenoviral vectors expressing single cytokines (IL-2, IL12 ) in which lymphocytes are attracted into the tumor but show no signs of activation (personal observations). The experimental results showing that tumors were rejected upon rechallenge suggest that infiltrating leukocytes could contain tumor-specific effector cells. In addition, mice who died from their tumors were observed to have multiple metastasis in their internal organs (lungs, liver, and spleen). Mice whose tumors had been rejected after vector administration showed no evidence of tumor metastasis upon sacrifice.

Importantly, this approach differs from those using soluble Abs (such as soluble anti-CD3 or bispecific Abs) therapy in several ways. In those applications, $\mathrm{CD}^{+}$cells are immediately coated with antibody after systemic administration of specific or bispecific Abs, causing the $\mathrm{Ab}$-coated lymphocytes to leave the circulation and traffic to lymphoid organs. ${ }^{24}$ Another limitation of bispecific antibodies is that antibody-coated lymphocytes exhibit only one cytolytic cycle, requiring the administration of excess antibody or infusion of large numbers of functional $\mathrm{T}$ cells with specific antitumor reactivity into patients in order to achieve good therapeutic efficacy. Moreover, purified antibodies are rapidly diluted upon injection and are likely to exhibit a very short half-life. ${ }^{25}$ T-cell activation by TCR - or CD3-specific, membrane-anchored antibodies, as expressed by the MVA vectors, is expected to be limited to tumor-infiltrating lymphocytes and thus is likely to reduce the potential toxicity of the treatment and the immunogenicity of the antibody.

$\mathrm{T}$ cells typically require two signals for activation - one produced by interaction of the TCR with its specific ligand, and the other generated by the interaction of co-stimulatory molecules such as CD80 or CD86 on APCs with CD28 on $\mathrm{T}$ cells. Whether the therapeutic effect of this approach can be increased by co-expression of these or other T-cell costimulatory molecules is currently under investigation.

There are several fundamental differences between RenCa and B16F10 that could account for the observed difference in rejection frequencies upon injection with the MVA-KT3 construct. B16F10 tumors grow much more quickly than do RenCa tumors, which may result in outgrowth of the tumor prior to an effective immune response. RenCa cells express MHCI, whereas B16F10 express little or no $\mathrm{MHCI}^{26}$ Growth of RenCa tumors has been shown to involve Tcell-mediated immune suppression. ${ }^{27-29}$ RenCa cells express ICAM-1, whereas B16F10 cells do not express ICAM-1 (our own unpublished data) and rejection of the tumor has been observed to depend upon expression of ICAM-1. ${ }^{30}$ These, as well as other possible differences between RenCa and B16F10 tumors, may account for the differences observed in effectiveness of MVA-KT3 in the control of tumor growth.

It has been shown that stimulation of $\mathrm{T}$ cells through the CD3 complex can differ from T-cell stimulation through the TCR itself. $^{23}$ Therefore, despite the ability of both to stimulate IL4 and IFNg equivalently, there may well be other cytokines or activities stimulated by the anti-TCR construct which are not, or less efficiently, stimulated by the anti-CD3 construct and vice versa. In view of the numerous differences between B16F10 and RenCa, as described above, this could have an impact on therapeutic effects of these two viral constructs as well as observed differences in the ability to induce effective immune memory

It has been reported recently that the intravenous injection of humanized soluble anti-CD3 antibody into cancer patients resulted in the resolution of malignant ascites in 3 
of 24 patients. $^{31}$ This observation, together with our own results, suggests that the expression of TCR-specific antibodies using an MVA vector may form the basis of a novel tumor therapy strategy especially suited for accessible tumors exhibiting various immune escape mechanisms. ${ }^{32}$

\section{Acknowledgments}

The authors sincerely thank K Dott and J Kintz for technical assistance, and $\mathrm{P}$ Slos and $\mathrm{P}$ Erbs for help in statistical analysis. This work was supported, in part, by the Convention Industrielle pour la Formation par la Recherche CIFRE.

\section{References}

1. Weijtens ME, Willemsen RA, Valerio D, Stam K, Bolhuis RL. Single chain Ig/gamma gene-redirected human T lymphocytes produce cytokines, specifically lyse tumor cells, and recycle lytic capacity. J Immunol. 1996;157:836-843.

2. Tibben JG, et al. Pharmacokinetics, biodistribution and biological effects of intravenously administered bispecific monoclonal antibody $\mathrm{OC} / \mathrm{TR} \mathrm{F}\left(\mathrm{ab}^{\prime}\right) 2$ in ovarian carcinoma patients. Int $J$ Cancer. 1996;66:477-483.

3. Chattopadhyay U. Tumour immunotherapy: developments and strategies. Immunol Today. 1999;20:480-482.

4. Nanni P, Forni G, Lollini PL. Cytokine gene therapy: hopes and pitfalls. Ann Oncol. 1999;10:261-266.

5. Meuer SC, et al. Antigen-like effects of monoclonal antibodies directed at receptors on human $\mathrm{T}$ cell clones. $J$ Exp Med. 1983;158:988-993.

6. Ellenhorn JD, Hirsch $\mathrm{R}$, Schreiber $\mathrm{H}$, Bluestone JA. In vivo administration of anti-CD3 prevents malignant progressor tumor growth. Science. 1988;242:569-571.

7. Liao KW, Lo YC, Roffler SR. Activation of lymphocytes by anti-CD3 single-chain antibody dimers expressed on the plasma membrane of tumor cells. Gene Ther. 2000;7:339-347.

8. de Ines C, Cochlovius B, Schmidt S, Kipriyanov S, Rode HJ, Little M. Apoptosis of a human melanoma cell line specifically induced by membrane-bound single-chain antibodies. J Immunol. 1999;163:3948-3956.

9. Paul S, Snary D, Hoebeke J, et al. Redirection of macrophage cytotoxicity using a non-replicative live vector expressing a tumor-specific scFv. Hum Gene Ther. 2000;11:1417-1428.

10. Xie YC, Hwang C, Overwijk W, et al. Induction of tumor antigen-specific immunity in vivo by a novel vaccinia vector encoding safety-modified simian virus $40 \mathrm{~T}$ antigen. $J$ Natl Cancer Inst. 1999;91:169-175.

11. Venkatesan S, Baroudy BM, Moss B. Distinctive nucleotide sequences adjacent to multiple initiation and termination sites of an early vaccinia virus gene. Cell. 1981;25:805-813.

12. Chen L. Overcoming T cell ignorance by providing costimulation. Implications for the immune response against cancer. $A d v$ Exp Med Biol. 1998;451:159-165.

13. Anilionis A, Wunner WH, Curtis PJ. Structure of the glycoprotein gene in rabies virus. Nature. 1981;294:275-278.

14. Mendiratta SK, et al. Intratumoral delivery of IL-12 gene by polyvinyl polymeric vector system to murine renal and colon carcinoma results in potent antitumor immunity. Gene Ther. 1999;6:833-839.
15. Tomonari K. A rat antibody against a structure functionally related to the mouse T-cell receptor/T3 complex. Immunogenetics. 1988;28:455-458.

16. Kubo RT, Born W, Kappler JW, Marrack P, Pigeon M. Characterization of a monoclonal antibody which detects all murine alpha beta T cell receptors. J Immunol. 1989;142 2736-2742.

17. Okada CY, Holzmann B, Guidos C, Palmer E, Weissman IL. Characterization of a rat monoclonal antibody specific for a determinant encoded by the $\mathrm{V}$ beta 7 gene segment. Depletion of $\mathrm{V}$ beta $7^{+} \mathrm{T}$ cells in mice with Mls-1a haplotype. J Immunol. 1990; $144: 3473-3477$.

18. Paul S, et al. Redirected cellular cytotoxicity by infection of effector cells with a recombinant vaccinia virus encoding a tumor-specific monoclonal antibody. Cancer Gene Ther. 2000;7:615-623.

19. Gajewski TF, Schell SR, Nau G, Fitch FW. Regulation of Tcell activation: differences among T-cell subsets. Immunol Rev. 1989;111:79-110.

20. Krutmann J, Kirnbauer R, Kock A, et al. Cross-linking Fc receptors on monocytes triggers IL -6 production. Role in antiCD3-induced T cell activation. J Immunol. 1990;145:13371342.

21. Zanders ED, Lamb JR, Feldmann M, Green N, Beverley PC. Tolerance of T-cell clones is associated with membrane antigen changes. Nature. 1983;303:625-627.

22. Norman DJ, et al. A US clinical study of Orthoclone OKT3 in renal transplantation. Transplant Proc. 1987;19:21-27.

23. Henrickson M, Reid J, Bellet JS, Sawchuk SS, Hirsch R. Comparison of in vivo efficacy and mechanism of action of antimurine monoclonal antibodies directed against TCR alpha beta (H57-597) and CD3 (145-2C11). Transplantation. 1995;60:828-835.

24. Weijtens ME, Hart EH, Bolhuis RL. Functional balance between $\mathrm{T}$ cell chimeric receptor density and tumor associated antigen density: CTL mediated cytolysis and lymphokine production. Gene Ther. 2000;7:35-42.

25. Takahashi K, Ono K, Hirabayashi Y, Taniguchi M. Escape mechanisms of melanoma from immune system by soluble melanoma antigen. J Immunol. 1988;140:3244-3248.

26. Banat GA, Christ O, Cochlovirus B, Pralle HB, Zoller M. Tumour-induced suppression of immune response and its correction. Cancer Immunol Immunother. 2001;49:573-586.

27. Gregorian SK, Battisto JR. Immunosuppression in murine renal cell carcinoma: I. Characterization of extent, severity and sources. Cancer Immunol Immunother. 1990;31:325-334.

28. Gregorian SK, Battisto JR. Immunosuppression in murine renal cell carcinoma: II. Identification of responsible lymphoid cell phenotypes and examination of elimination of suppression. Cancer Immunol Immunother. 1990;31:335-341.

29. Cavallo F, Martin-Fontecha A, Bellone M, et al. Coexpression of B7-1 and ICAM-1 on tumors is required for rejection and the establishment of a memory response. Eur $J$ Immunol. 1995;25:1154-1162.

30. Kufer $\mathrm{P}$, et al. Construction and biological activity of a recombinant bispecific single-chain antibody designed for therapy of minimal residual colorectal cancer. Cancer Immunol Immunother. 1997;45:193-197.

31. Richards J, Auger J, Peace D, et al. Phase I evaluation of humanized OKT3: toxicity and immunomodulatory effects of hOKT3gamma4. Cancer Res. 1999;59:2096-2101.

32. Gilboa E. How tumors escape immune destruction and what we can do about it. Cancer Immunol Immunother. 1999;48:382385 . 